\title{
El Conflicto Organizacional de la Educacion del Management Brasileño bajo la Visión del habitus "Bourderiano"
}

\author{
O Conflito Organizacional da Educação do Management Brasileiro sob a Perspectiva do \\ habitus"Bourdieusiano"
}

Cristian Eugenio Orellana Pino ${ }^{1}$

\begin{abstract}
Resumen
Este analisis es un ensayo critico-teórico sobre el estudio de los campos organizacionales que indagan sobre conflictos de auto-intereses que convergen entre los sistemas organizacionales y los referentes constructos de Bourdieu (1983). Se desarrolla un modelo conceptual tríadico de integración de representación entre el campo de la Universidades, el Habitus Bourideriano y las expresiones del Ethos. Es un ensayo de crítica al management hacia sus intereses egoístas (propios intereses) que están enraízados en la academia, comprendiendo la naturaleza peculiar (cinismo) del hacer un estado del arte fetiche la gestión en las empresas, como estudios organizacionales (narcicismos), conceptos que transformaran sus elementos gerencia blesen instrumentos fetiches (vanidades).
\end{abstract}

Palabras-Clave: Convergencias. Ética. Habitus. Universidad. Campo Organizacional.

\section{Resumo}

Esta análise é um ensaio critico-teórico sobre o estudo dos campos organizacionais que indagam a respeito dos conflitos de interesse que convergem entre os sistemas organizacionais e alguns modelos teóricos do campo de Bourdieu (1983). Desenvolve-se um modelo tríadico de representação conceitual entre o campo da Universidade, o hábitus Bourdieusiano e as diversas expressões do Ethos. É uma critica à formação do management com foco nos interesses egoístas (próprios interesses) que traz da academia, ao observar sua natureza particular (cinismo) do fazer um estado da arte fetiche a gestão das empresas, estudos organizacionais (narcisismo), conceitos que transformam seus recursos gerenciais em instrumentos fetiches (vaidade).

Palavras - chave: Convergências. Ética. Habitus. Universidades. Campo Organizacional.

\footnotetext{
Artigo submetido em 19 de agosto de 2013 e aceito para publicação em 03 de julho de 2014.

DOI: http://dx.doi.org/10.1590/1679-39518943

Ingeniero em Administración de Empresa; Licenciado em Gestión de Empresas, Facultad de Economía y Empresa, Universidad Diego Portales UDP Chile; Magister en Marketing, Facultad de Economía y Negocios Universidad de Chile. Dirección: Calle Regina Paci, 760, Nuñoa, Santiago, Chile: E-mail: cristian1914@hotmail.com
} 


\section{Preambulo al Modelo Convergencias}

Este análisis es uno estudio crítico mediante el empleo de un modelo de convergencia que esquematiza la formación del management en Brasil mediante los constructos del habitus de Bourdieu. Constituido por un proceso de formación heurística, con diversos constructos como el habitus Bourderiano en lo que respecta a la naturaleza de formación de las escuelas de negocios en universidades particulares en la región del noreste de Brasil.

El marco esquemático de representación se compone de constructos de la siguiente manera: el papel de las escuelas de negocios (organizaciones privadas lucrativas) que cumplen una función como agentes racionales y sin el componente de incorporar la formación humanística como se piensa actualmente, ya que estas responden a sus propios intereses del mercado. Dando espacio al narcicismo académico que impera (educación napoleonica) y no a los intereses de la sociedad (educación humboldiana) ambos incorporados al sistema educación mediante el proceso histórico de formación. Si bien es parte de un egoísmo de ámbito económico que prevalece para la formación de las escuelas de negocios.

Lo anterior se asienta en un argumento del conflicto que genera los juegos de intereses. Que ciertamente están relacionados por algo común que comparten y se transfieren por un habitus y por otro lado intereses que divergen debido a egoísmo económico que representa cada escuela de negocio en el modelo de universidad-empresa. Que modifican sus Ethos como los juicios de valor. Pues también mediante este habitus se puede plantear un cuestionamiento acerca de las decisiones como opciones estratégica sobre qué conveniente de lo otro. Ademas como recurso apoya a comprender qué es lo que provoca la divergencia de relaciones entre la firma o universidad-empresa y la formación del profesional en las escuelas de gestion. Por ejemplo, universidades particulares, luchan por captar alumnos, recursos, infra-estructura, posicionamientos, preferencias, etc.

Para una didactica pedagógica de entender el modelo, se presenta un esquema como mapa conceptual con los constructos triadicos de manera alcanzar una mejor comprension del contenido conceptual de este análisis (ver Figura 1).

Figura 1.

Modelo Convergente de Interés

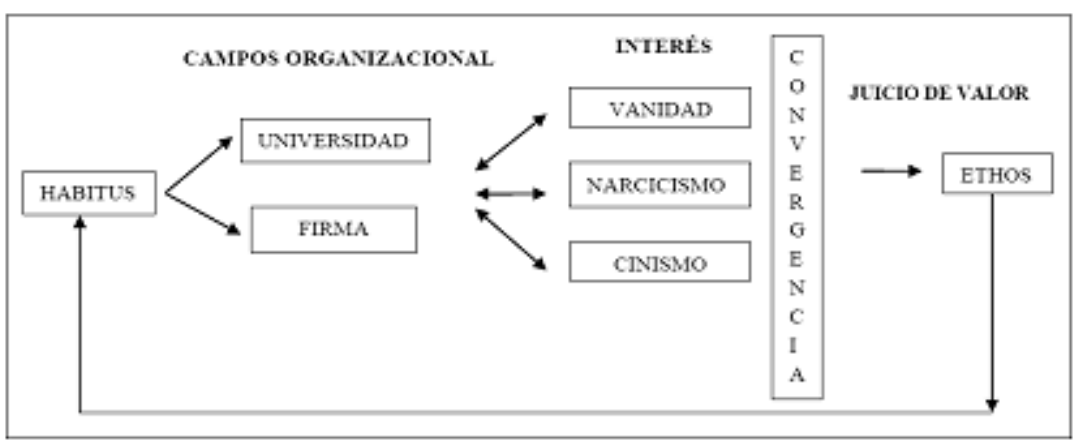

Fuente: Elaborado por el autor.

Con respecto al mecanismo de cómo se constituyen las escuelas de negocios en la región del Noreste de Brasil esta asentado sobre la visión del lucro de interés económico de sus agentes. Que se distorsiona cuando incorporamos las diferentes expresiones del Ethos como dimensiones del habitus en las escuelas de negocios.Y que luego se plasman en los contenidos y la naturaleza de la formación de la malla curricular en prode la formación del profesional. Colocandolo como una representación hacia un discurso ético de relacion de buenas acciones. 
De lo anterior, se puede decir que la formación de la malla curricular se debe a que en las directrices de estos tipos de organizaciones privadas o escuelas de negocios sientan sus bases en el poder de las relaciones que se tornan mutuas y afiatamiento con las empresas para esta región. Con un foco dominante de orientar estudiantes como a docentes a formar individuos según las características representativas con el perfil de estas universidades-empresas. Desprovisto del entendimiento de la formación humanista y que luego se posicionan en el mercado reproduciendo las características simbólicas de quienes las transfieren (NAVARRO, 2006).

\section{Datos del Sector Universitario}

Según el último censo del 2004 realizado sobre la Educacion Superior en Brasil, habian 2.013 instituciones de educación superior que impartian cursos de graduación, donde el mayor aumento de entidades de acuerdo a los años anteriores estaban en las regiones Norte (16.8\%) y Nordeste (13.2\%). Habiendo en la región del nordeste un aumento de 40 nuevas instituciones. En 2006 habian 4.7 millones de alumnos estudiando en las universidades, $74 \%$ estaban concentrados en el sector privado. El sector con fines lucrativos concentraba 41 $\%$ de los registros de matricula, en contraste el sector publico con $25 \%$, habiendo aumentado el numero a 2.270 entindades, donde $89 \%$ estaba concentrado en sector privado, de estas un $78 \%$ pertenecian al sector con fines lucrativos (MARTINS, 2009).

La Tabla 1contiene el ritmo del crecimiento de organizaciones donde se indican que el sector privado durante el año 2004 tuvo un aumento de $8.3 \%$ con un incremento de 137 nuevas entidades. Según este Censo indican que hubo un menor registro de acuerdo al año 1997. En un universo educacional de pre-grado, el mayor grupo es el sector privado que representa el 88.9\% del total. Al año 2004 laregion del Noreste reunia 344 instituciones. Hoyel ritmo de crecimiento de nuevas instituciones de educacion superior privado ha decrescido, esto se observa por un salto de $24.2 \%$ del año 2002 a $14.3 \%$ al año 2004.

Tabla 1

\section{Crecimiento de entidades a nivel nacional}

\begin{tabular}{|r|r|r|r|r|r|r|}
\hline \multicolumn{7}{|c|}{ Crecimiento de las IES por Región - Brasil 1990 - 2004 } \\
\hline Ano & Brasil & Norte & Nordeste & Sudeste & Sur & $\begin{array}{l}\text { Centro } \\
\text { Oeste }\end{array}$ \\
\hline 1990 & 918 & 26 & 111 & 564 & 146 & 70 \\
\hline 1995 & 894 & 31 & 92 & 561 & 120 & 90 \\
\hline 2000 & 1180 & 46 & 157 & 667 & 176 & 134 \\
\hline 2004 & 2013 & 118 & 344 & 1001 & 335 & 215 \\
\hline
\end{tabular}

Fuente: MEC/Inep/Deces

A modo de cararectizar el perfil de la educación superior en el pais, la Tabla 2 presenta un total de 2.013 de entidades que según la legislacion vigente que normaliza la creacion de nuevas instituciones existen 169 Universidades; 107 Centros Universitarios; 119 Facultades Integradas; 1.474 Facultades escuelas e Institutos y 144 Centros Tecnologicos. 
Tabla 2

Distribución de organizaciones académicas

\begin{tabular}{|l|r|c|}
\hline \multicolumn{3}{|c|}{ Instuciones de Educación Superior } \\
\hline & \multicolumn{1}{|c|}{ IES } & $\%$ \\
\hline Universidades & 169 & 8.4 \\
\hline Centro Universitarios & 107 & 5.3 \\
\hline Facultades Integradas & 119 & 5.9 \\
\hline Facultades, Colegios e Institutos & 1.474 & 73.2 \\
\hline $\begin{array}{l}\text { Centro de Educación Tecnológica } \\
\text { y Facultades de Tecnologia. }\end{array}$ & 144 & 7.2 \\
\hline \multicolumn{1}{|c|}{ Total } & 2.013 & 100 \\
\hline
\end{tabular}

Fuente: MEC/Inep/Deces

Tabla 3

Distribución y Crecimiento de entidades en la región Noreste

\begin{tabular}{|c|r|r|r|c|c|c|}
\hline \multicolumn{7}{|c|}{ Distribución de las IES en la Región Nordeste - Brasil 2001 - 2014 } \\
\hline Ano & Brasil & Nordeste & Publico & $\%$ & Privado & $\%$ \\
\hline 2001 & 1.391 & 211,00 & 46 & 21.8 & 165 & 78.2 \\
\hline 2002 & 1.637 & 256,00 & 51 & $19 . .9$ & 205 & 80.1 \\
\hline 2003 & 1.859 & 304,00 & 52 & 17.1 & 252 & 82.9 \\
\hline 2004 & 2.013 & 344,00 & 56 & 16.3 & 288 & 83.7 \\
\hline
\end{tabular}

Fuente: MEC/Inep/Deces

De acuerdo la Tabla 3 em la región Noreste el sector privado abarca un $83.7 \%$ yse distribuye en diferentes tipos de organizaciones, ya el sector publico alcanza un $16.3 \%$ de 344 entidades para esta region. ¿Qué es lo que ocurre en ambos sectores en una región que ocupa 1/3 del terriorio nacional?. Vale destacar que el sector tecnologico tuvo el mayor crecimiento de todos los modelos de organizacion academica. Es interesante notar que existe una mayor preferencia por carreras tecnologicas, sea por la menor duración, menor carga academica en la malla curricular o una salida laboral inmediata.

Consta que else ha creado una idea que en a un corto periodo de aprendizaje acarrea el oportuno crecimiento de organizaciones privadas delegando una diversidad de preferencias por carreras de corta duracion. En la Tabla4 se observa que al año 2004 la mayor proporción de cursos de pré-grado en todo el pais se concentra en la carrera de escuelas de negócios alcanzando um número de matriculas de 620.718 alumnos, esto conlleva a um $14.96 \%$ del total de la carreras de formacion profesional.

Tabla 4

Orden de la Demanda de carreras de pre-grado a nivel nacional.

\begin{tabular}{|l|r|r|r|}
\hline \multicolumn{4}{|c|}{ Los 10 Mayores Cursos por Numero de Matriculas y Egresados } \\
\hline Cursos & Matricula & $\%$ & Egresados \\
\hline Administracion & 620.718 & 14.9 & 83.659 \\
\hline Derecho & 533.317 & 12.8 & 67.238 \\
\hline Pedagogia & 388.350 & 9.3 & 97.052 \\
\hline Ingenieria & 247.478 & 5.9 & 23.831 \\
\hline Letras & 194.319 & 4.7 & 37.507 \\
\hline Comunicación Social & 189.644 & 4.6 & 26.816 \\
\hline Contabilidad & 162.150 & 3.9 & 24.213 \\
\hline Educacion Fisica & 136.605 & 3.3 & 17.290 \\
\hline Enfermeria & 120.851 & 2.9 & 13.965 \\
\hline Computacion & 99.362 & 2.4 & 13.601 \\
\hline Total & 2.692 .794 & 64.7 & 405.172 \\
\hline Brasil & 4.163 .733 & 100 & 626.617 \\
\hline
\end{tabular}

Fuente: MEC/Inep/Deces 


\section{Lo referente del habitus}

El habitus como la teoría de campo conceptual de Bourdieu (2006) es la referencia terminologica para este análisis, que permite realizar una descripción aplicada de quienes son los agentes y sus objetos, empleando los campos de relacionamientos sociales (entre objetos objetivados y agentes sustantivados). Donde la dinámica de acción es el posicionamiento de cada agente estar bajo un juego de intereses de objetos mobiles a través de un proceso histórico donde se transfieren en un proceso continuo caracteres implícitos de cada agente.

El habitus del campo social es regido por leyes establecidas que se modifican de acuerdo a un proceso de transformación histórica que revelan la casuística de los conflictos. Creando un juego de auto-intereses entre agentes dominados porsentimientos naturaleza moral (cinismos, narcisismos, vanidades).La propuesta de este análisis es revelar del constructo habitus las informaciones que se transfieren desde un conocimiento adquirido y que van trascendiendo. Por ejemplo, las practicas narciscistas propias de cada escuela de negocio como relaciones simbólicas de producción. Unarelacion directa con el universo del estudio cientifico filosofico, que tiene replicas en las costumbres y habitos tradicionales (BOURDIEU, 2010; NOSELLA, 2008). La función que constituye el Ethos en estecampo es evaluar cuales son las características comunes en esta región que induce determinan qué juicios de valor constituyen la caracteristicas morales de sus costumbres. Por ejemplo, las decisiones que orientan la dirección y las acciones de las personas pose en una carga de juicios de valorque hace posible el poder o no guiar una tarea y que potencie el ejecutar la o no. El tomar la familia la decisión de optar el ingreso por alguna escuela u otra mejor en un ranking.

De lo anterior se puede substraer la conductay prácticas sujetas a la transferencia delos juicios de valor enraizados en la producción simbólica cultural y economica de la región. Se observaque las escuelas de negocios de universidades particulares en esta región incorporan una escasa la terminología Etica en los contenidos de la formacion del management. Aquí el dominio y poder delinteres de los agentes en las escuelas de negocios es mayor por entregar conocimiento vinculados a una formación de cuerpo tecnico que demandan las firmas.

La relación que cabe a la introducción del campo de Bourdieu es hacer una relación de cómo se han reforzado los lazos de los agentes y han desarrollado una transferencia de informaciones permanentes a través de agregaciones de subcampos que ha hecho fortalecer un sistema educacional privado como una estructura común. Cada campo posee elementos que capitalizan socialmente y convergen por intereses que hacen de un proceso la creación simbólica de un campo organizacional de convergencia de intereses.

El análisis crítico hacia este modelo educacional de formación del management, es sobre un fenómeno en particular que prospera en las escuelas de negocios qué elementos permiten la reproducción continua de por ejemplo, la satisfacción que otorga el lucro para conseguir una mejor posición en el mercado educacional, ranking que permite promoción aumentar las matriculas, crecimiento de numero de campus y ampliar las carreras de negocios, orientando y negociando la formación profesional hacia un sistema frenético que es el mercado. O es una manifestación del propio Ethos particular de esta región.

De lo anterior, se produce la utilidad del modelo convergente, al visualizar las relaciones de interés de acuerdo al contexto a compartimientos de sus campos. Al revelar entre los elementos constituyentes del Ethos, sus componentes - cinismo, narcicismo y vanidad -. Los coloca en situaciones que convergen y divergen. De lo anterior surge el conflicto de interés entre los elementos constituyentes y su relación que comparten en común los campos de estudio. Así como se pretende substraer informaciones y demonstrar acerca de cómo las escuelas de negocios en universidades privadas en esta región, potenciansus propios interesesy aumentan cada vez su capital economico.

Ciertamente para este caso, constituyese el habitus para producirun analisis de creacion de organizaciones formales en un contexto de formación pedagogica-laboral donde individuos cargan sus percepciones y comportamientos de cambios de conducta. Conjunto de disposiciones que se adquieren en un contexto 
particular de un momento social particular, como reproducción de nuevas practicas, de acuerdo a la dominancia y posición en cada contexto.

Si bien este construto de los campos es la pieza que articula y hace posible que el mecanismo del habitus este presente, el ethos con sus caracteres de producción simbolicay la universidad-empresa como medio dominante. Además que permite visualizar la naturaleza de la accion de las ideas y la representación a traves de las prácticas externas sociales. Según Bourdieu (1990), esta manera de analisis relacional solo es posible entre lo que es permitido en una institucionalidad como via de regla y sumanera de improvisarel cotidiano.

La realización de adquisición de aprendizajes por causa de diferentes disposiciones de juicios de valor, se adaptan a lo irregular que suele suceder muchas veces lo imprevisto. Esto tiene un sentido simbolico (BOURDIEU, 1998) si lo que se pretende desarrollar es un aprendizaje de las diferentes percepciones que puede tener las actitudes del Ethos en la practica diaria dentro un ambiente organizacional donde los codigos y las indeterminaciones en las divergencias que pueden ser las situaciones improvisadas, por ejemplo en el area de negocios, el riesgo a la toma de decisiones, la incerteza de acciones no previsibles, los horizontes futuros, los pronosticos de presupuesto, el entorno externo del negocio, las imperfecciones de la planificación, etc.

El modelo simbolico que representa el contenido del Ethos para habitus organizacional para un contexto de alumnosen proceso de formación de su aprendizaje permite entender la naturaleza en este contexto la fuerza de su integración social. Produccion de conocimiento y sus códigos de comunicación, que hace más real sus percepciones sobre su campo social y constribuye para la reproducción del orden social. Para el caso de las escuelas de negocios la producción de conocimiento con tendencia a su justificación ética, parece estar relacionado a lo que Weber (1981) se refiere a que toda acción que se atesora decir ética es porque posee un doble discurso que convergen entre si, dicha afirmación es orientar se a una ética de la intención o hacia una ética de la responsabilidad. Y es esto ultimo que ha generado la convergencia de interés para la actitudes que el Ethos incorpora.

Hay que constatar que este poder simbólico puede ser mal interpretado cuando hayun abuso conciente de realizar un discurso sobre las practicas éticas, principalmente cuando no representa y no es reconocido por los grupos de interés. Por ejemplo, significa instaurar un poder del capital economico a modo de justificar y aceptar la logica de cada negocio universidad-empresa. Alterando las reglas de conducta, las disposiciones adquiridas como capital de distinción de luchas por lograr el reconocimiento por espacios conscientes de sus representaciones sociales como esfuerzo de superación de su realidad y percepciones, siendo transformadas constantemente, estos es, la producción de las divergencias que genera el campo social universidad-empresa.

Esta constatacion es vista cuando se intenta delegar a un segundo plano las relaciones de poder que se instauran en cada espacio. Como corrientes sociales que separan la construcción epistemológica del constucto del Ethos en ambientes organizacionales en sistema de procesos continuos ingresando informaciones pre-diseñadas de objetos, reduciendo todo un tejido social (BOURDIEU, 1990; 1994; 2000). En este sentido el concepto de habitus se va alienando un término estrategico que Bourdieu (1990) suele distinguir como el senso practico que posee el habitus que va orientando nuestras acciones en continuidad con las estrategias internas propias a las determinadas por la sociedad, los individuos capaces de subvertir las normas vigentes de una sociedad, según las practicas individuales.

Por ejemplo, para individuos que estudian en espacios marginalizado la pobreza es un indicador no menos importante sino determinante para el suceso de una vida profesional, o justificativa para salir de impase de falta de expectativas futuras o enfrentarse con su cotidiano (PIOTTO, 2008; LAHIRE, 1997; BOURDIEU, $1983 ; 1989 ; 2005)$.

En este punto de partida es donde la estrategia del juego de intereses se desprende como sentido de orientación hacia prácticas que reproducen sus realidades o como un juego de modas u objetos simbolicos (BOURDIEU, 2000; 2007). Por ejemplo, algunas universidades privadas en conjunto con las políticas de las escuelas de negocios incorporan nuevas carreras como objetos fetiches ajeno al cotidiano del habitus de cada 
alumno. En este sentido el confronto entre ambos tipos de representación social y sus relaciones de poder, como indica Bourdieu (2007) traen como linea de inflexión las divisiones sociales, por ejemplo, que primero se componen de aquellas que son comunes en escuelas de negocios de acuerdo a los contenidos universales que demanda el entorno externo, a decir, generar una producción homogenea de creencias y contenidos pedagógicos en todas las escuelas de negocios, y segundo, aquel que divide la sociedad de acuerdo a criterios de producción perceptivas como aquellos juicio de valor que llevan a entender cual es la mejor orientación que conduce al bien común.

\section{El aporte de los constructos a los Campos}

El aporte de las universidades del sector privado con sus escuelas de negocios, particularmente lo que concierne el aporte a sectores de comunidad locales ha sido poco estudiado en Brasil, sin embargo, se logra identificar los aportes en cuanto a los proyectos que generan impactos económicos y sociales, orientado a un tema referente al Ethos que es la responsabilidad social como fuerzas de sus relaciones comunes.El punto de continuidad con el habitus es entender como las universidades particulares miran dentro de sus escuelas de negocios hacia estos temas con respecto en adoptar enfoques que les aproxime a las comunidades locales y hacer frente a problemas de sus grupos sociales.

Un estudio de Glasson (2003), Robinsony Adams (2008), verificaron que la atención que se dá a estos problemas puede estar en la definición del rol que cumplen y reputación institucional de organizaciones educacionales (reputación, influencia, tamaño, etc.), el modo en como se envuelven sus iniciativas y se tornan agentes activos en la sociedad, haciendo frente a barreras sobre retorno de beneficios y ganancias de comprometen dichas iniciativas.

En esta línea, el contexto de impactos que producen y pueden generar la dimensión social, cultural y político el aporte de las universidades al desarrollo de comunidades locales, cuanto a nuevos tipos de aportes no solo económicos sino además contribuir hacia mejorar la percepción de sus relaciones que se tienen de éstas. Para Robinson y Adams (2008) estas contribuciones permiten mejorar la imagen de las organizaciones por medio de proyectos de sumen aportes significativos hacia las comunidades. La anterior visión aunque parece ser bastante optimista, sin embargo, posee una doble lectura, según Calderón (2000), para el sistema de educación universitario existe una noción del ciudadano cliente-consumidor, mercado-de-educación, que alteran la realidad del sistema universitario en el sector privado - universidades mercantiles - que le otorgan una fluidez al mercado, debido a su lucratividad y competencia donde suelen inserirse estas organizaciones con fines de lucro. Que ofrecen productos y servicios de alta demanda (por ejemplo, las formaciones en carreras de negocios de corta duración, alianzas con bancos indicados para otorgamiento de créditos de estudios, etc.).

Estas empresas educacional es mantien en un discurso que revela infinidad de nuevos conceptos y objetos, una renovación de significados, que además abordan temas de acuerdo a las categorías de cada segmento educación-empresa. La Ley de Directrices y Bases de Educación (LDB), en 1996 alteró el programa de apertura para nuevas entidades educacionales de nivel superior, otorgando mayor autonomía a estas. Alterando el panorama para la formación de universidades-empresas, que regula en la práctica potencializar cursos de mayor demanda, alterando el número de vacantes según el flujo de evasión, decisiones que se ajustan a las fuerzas del mercado. Este encuadramiento orientado al mercado deja evidente un prospecto diversificado de productos y servicios educacionales, cómo acentuar la diferenciación entre escuelas de negocios que compiten por cuotas de mercado ¿cliente-consumidor?

¿Cuál naturaleza intrínseca les hace regular sus programas educacionales hacia una alta o baja demanda?, como la cuota de retorno que obtiene por el nivel de inversión en capital, ¿sonestos retorno garantizados que se dan a los cursos que presentan grande demanda y bajo costo de inversión financiera? Resultando en cursos de bajo costo por mayor volumen de estudiantes?, Esta operacionalidad de sus activos está en función de 
mantener profesores en función de régimen parciales de contratación?, panoramas como estos son propios de universidades mercantiles, donde estas organizaciones acaban no generando vínculos sociales sino divergencia debido a sus interés particuales de cada escuela de negocios.

El empobrecimiento de la calidad educacional, puede o no alterar el capital social enfocado a los contenidos, sin embargo, no garantiza el logro final de los individuos. En esta región, gran parte de las escuelas de negocios de establecimientos universitarios es privado, con una oferta de cursos no tan diversificados al constatar se una oferta mayor tanto de carreras como de vacantes, parece indicar que un valor reducido de carreras permite al individuo tener mas opciones y preferencia por estos llamados curso en masa.

Mirando hacia un nivel superior de las escuelas de negocios, para Bertero (2007); Fisher (2001); Oliveira (2006) hay un enorme inter és y motivación que movilizan a estudiantes a optar por hacer carrera sy programas de pos-grado en el ámbito de la gestión, sea por el impulso que el clima socio-político favorable al crecimiento y desarrollo económico del país y la expansión de los programas americanos de posgrado y especialización en USA han contribuido al diseño de este escenario. Los autores resaltan además que existe una necesidad de modernización del Estado y desarrollo país.

En este último punto Wood y Paula (2004) los programas de intercambio docente entre USA ha sido un impulso que promueven un aporte y mejora a la formación del cuerpo docente en Brasil. Si bien existe un problema de estructuración de los establecimientos como de los contenidos de los programas de gestión que atiende a una pequeña elite económica y cultural al considerar los centro de mayor prestigio. Con el tiempo seha creado un desorden debido a la ausencia de reglas de calidad y validación del desempeño de las escuelas de negocio. Para Oliveira $(2004 ; 2005 ; 2006)$, problemas como jugar con la demanda y la multiplicación de escuelas aisladas y facultades con poca calidad generó la creación de normas más rígidas y el cancelamiento de nuevos registros para apertura de nuevos campus. Con relación a esto, el crecimiento de establecimiento a un nivel histórico se estagnó en la década del 70, para aumentar exponencialmente el número de matriculas en entidades privadas en la década 90 de esta manera lograr satisfacer la demanda para estos programas de gestión. Los cursos de gestión en Brasil a modo general superan cualquier carrera de pregrado, el número de establecimiento alcanza el orden de 1.734; y los programas cuentan con 123.000 estudiantes de posgrado.

En Brasil, el acercamiento hacia el mercado de consumo de la educación superior de modo general, se debe a un proceso de herencia histórica que tiene sus bases en la universidad francesa tecnicista, que se contra restracon el proceso del surgimiento de la universalidad de base neo-humboldiano que promueve un distanciamiento del mercado por una aproximación hacia el conocimiento erudito(SGUISSARDI, 2002; MOLLIS y MEEK, 2002; SCHUGURENSKY, 2002). Se ha creado una matriz profesionalizante donde la oferta de las escuelas de negocios solo existe para ofrecer una educación profesional muy temprana, adquiriendo una visión del mundo restricta, antes de ser y adquirir conocimientos, dejando de lado una formación más extensa orientada al universo humanista, histórico y social (NUNES y DE CARVALHO, 2007).

Si bien la formación de cuadros profesionalizante es un legado de los campus franceses de la especialización delas carreras. Hay que resaltar que la grande influencia que ejerce una fuerte presión por alterar del contenido de los programas en las escuelas de negocios proviene de las corporaciones profesionales. Ya que estas participan en la deliberación indirectamente en la decisiones de los contenidos de dichos programas de negocios, integrando las demandas por profesionales técnicos de acuerdo a la estructura de oferta de cursos (Edital 04/97 Comisión Especial SES u MEC). Particularmente esto es bien observado en las escuelas privadas de gestión donde según el perfil profesional que demanda la sociedad, se crea una educación superior asociada mucho más orientada a una vida corporativa que a una vida educacional.

Es necesario indicar para qué la educación es necesaria y hasta qué nivel de desarrollo es elemental y si representa una preocupación en satisfacer los fines sociales. No deja de ser una preocupación, sin embargo, suele parece que existe un cierto productivismo que solo esta preocupado por medir el suceso universitario. Una aproximación relativa a esta línea es sobre la reflexión del espíritu crítico que se logra cuando ocurre el 
contraste entre la realidad cercana y las percepciones que se tienen de esta de manera endógena. Incorporar una actitud pedagógica que mude las creencias sobre una cultura educacional, que logre una postura reflexiva acerca del conocimiento, sólido argumento que incorpora la educación crítica en las escuelas de negocios.

Si bien hay una crisis de orientación institucional en las escuelas de negocios que apunta a la importancia que se le ha dado a las teorías y metodologías estadísticas a las carreras de negocios, perjudicando la formación de habilidades que ciertamente son necesarias al pensamiento crítico, como indispensable a la práctica y tomada de decisiones. En esta argumentación Fisher (2001) apunta hacia una crisis en la estructura curricular con base en el manejo de un concepto tradicional, donde este debe ser visto como medio para alcanzar la formación profesional que refleje modos y medios de aprendizaje, que visen el desarrollo de competencias socialmente deseables.

\section{Las fuerza dominantes de convergencia: Interés, Vanidad, Cinismo y Egoísmo}

De acuerdo a la tomada de decisiones motivado según su propio interés, el individuo emite dos tipos de juicios que según Almeida (1997) quienes emiten los juicios lo hace de dos formas, de acuerdo al juicio de valor y juicios reales siguiendo una línealógica (BURIASCO, 2007). Donde el juicio real se relaciona al tipo de evaluación que realiza cuando el individuo es sometido a un proceso de instrucción y control, que obtiene como resultado un valor lo más cercano a la realidad. Otorgándo le un significado a lo que el determina entender acerca de la realidad y no lo que el vivencia o experimenta (valor). Para el sistema de educaciónaprendizaje en las escuelas de negocios, los procesos de evaluación son colocados en función de la utilidad que logran satisfacer a quienes pretender substraer beneficios a expensas de un sincero proceso de instrucción de aprendizaje.

En los utimos años el proceso de aprendizaje atraviesa cambios significantes, especialmente, con el individuo que desea modificar y crear conocimiento y dejar de lado aquel que reproduce la información. Esta concepción de transformación deja de lado el proceso de reproducción. Sino que lo hace a través de un proceso de construcción y un acto de creación. Que códigos emplea el educador profesional en las escuelas de gestión y qué tipo de naturaleza lo conduce a desarrollar los significados en cada momento, recrear y resignificar los a todo instante.

El acto de vanidad obstruye y crea impases frecuente entre el individuo y el objeto de análisis. Este debiera utilizar sus propios recursos, basado en la subjetividad y su conocimiento aprendido. Y no sobreponerse por su interés sobre el colectivo. Es cierto que en situaciones de decisiones pueden basarse en reflexiones que tienen una carga de sabiduría informal, tomando actitudes inmediatas para resolver problemas, como un sistema de creencia, puntos de vista, costumbres, que muchas veces no obedece a teorías de aprendizaje que sin duda orienta éticamente al individuo a realizar sus acciones con base en sus propios principios e intereses personales. Sin embargo un agregado de altruismos lo expone a un exceso de vanidad individual. Larelación con sus intereses está en función de lo que es percibido por su alteridad con el otro.

Estas relaciones se ven obstruidas sea por distorsión que motivan, disciplinan y orientan el comportamiento humano en ciertas circunstancias, sea por la existencia de ambientes propicios para el desarrollo de espacios de diálogo. Se cree que es la existencia y la necesidad del otro la que moviliza la transferencia de conocimiento a través de un proceso de agregaciones. Para Solomon (1995) existe una microetica que aborda la naturaleza de las relaciones, las buenas intenciones, sus consecuencias e implicaciones y responsabilidades que deben responder a sus acciones entre dos individuos.

Las conductas deben ser entendidas de acuerdo a la particularidad del momento, llevando en consideración los actores con sus particularidades. Pensar acerca de la vanidad como es percibida en los programas de negocios, toma en consideración el ejercicio en cual se ven envueltos los individuos, el cambio que se produce acaba tornando responsable las actitudes y por consecuencia la autonomía de dirección que orienta 
el aprendizaje. Tornándola una práctica meramente psicológica como también una cuestión ética, reconociendo la individualidad que deben ser educados para valores del colectivo (OLIVEIRA y DOS SANTOS, 2005). El reconocer las diferencias y existencia de un otro agente y diferente del referencial.

Esto permite concluir que la comunicación entre ambos es posible, el compartir percepciones diferentes acerca de un mismo objeto es un aspecto que se debe considerar cuando me refiero a la ética en un campo social como las escuelas de negocios en universidades particulares.En un cuadro ético se requiere una posición frente a la realidad que en su cotidiano se despliegan acciones personales y colectivas, legítimas y que sean justificables. ¿Hasta qué nivel los valores son legítimos?, si existe una sociedad excluida de sus derechos, ¿es legítimo constituir un cuadro ético?. De modo a identificar una sociedad modelo que aspira al desarrollo de grupos socialmente organizados es necesario enfrentar el paradigma.En el ámbito de las prácticas de colaboración entre empresa y universidad, observamos que hay una ausencia de educación cívica mínima que caracterice a los ciudadanos, sea por lo que se observa en defectos hacia un modelo institucional fallido de la educación, desconocer los derechos y deberes, actitudes y comportamientos por paternalismo, autoritarismos, presunciones, egos, desequilibrios emocionales, es la evidencia de una vanidad institucional.

En un ámbito profesional, es evidente detectar oportunismo y competición. Introducir asignaturas en escuelas de negocios que por su mal manejo producen prácticas de gestión abusivas y perversas (superinflación en libros contables; burbujas inmobiliarias; estrategias de marketing persuasivas) haciendo de proyectos educacionales de formación, abandonar el saber a favor de presumir el dominio teórico hermético. Pretensiones que orientan a individuos adoptar una visión y criterios de imposición, prácticas que empresas fomentan por medio de un criterio impositivo y no por el diálogo que busca comunicar el discurso del bien común. Si bien la vanidad en la firma suele estar vinculada a los incentivos que poseen algunas empresas de manera a persuadir la búsqueda de ganancias por terceros, inducen al individuo a maximizar u optimizar su acciones; para Jensen (2008) e sinconcebible pensar que el propósito de una acción siendo humana pueda ser vista como alguna cosa que responde a incentivos. Imposición docente que universidades promueven de manera a que individuos adopten posturas que internalizan visiones reduccionista y estrictas de determinados autores y docentes.

La falta de credibilidad en organizaciones ha apuntado a que este fenómeno corresponde a diversos motivos que se cruzan por el camino de la ética, algunas causas comunes son: conflictos de interés, marketing y publicidad deshonestas, mala calidad de los productos, mal relacionamiento con la comunidad, comportamientos dudosos en las negociaciones colectivas laborales y comerciales; falta de transparencia; desconfianza en las relaciones entre colaboradores. La introducción de sistemas de control permite entregar incentivos e informaciones dentro de un contexto de decisiones con alto nivel de comprometimiento en conflictos éticos.Cohen, Pant y Sharp (2000) orientan a promover la importancia de efectuar análisis de asimétricos de informaciones, de forma a comprender qué es lo que estimula la promoción de incentivos con base en la premiación de utilidades, análisis de colaboradores; el impacto de individualismos ético en el comportamiento organizacional.

La participación es cuestionable cuando en la tomada de decisiones los cambios de comportamientos no se ajustan a la disposición de responsabilidades éticas. Una propuesta de una ética solidaria (CONILL, 2000); de integración empresarial (OROZCO, 2005); y pedagógica Paladino (2005). Es el consenso de llegar a un término que se ajuste a la ausencia del bien común, que el egoísmo ético produce en el entorno laboral y en el medio que gobierna el universo académico con la producción de científica.

Para el consejo internacional de la AACSB $(2004 ; 2005)$, señala que existe una crisis en la ética de los negocios y que este no solo se encuentra en tan solo cambiar las actitudes que asumen las firmas sino en aprovechar la oportunidad de fortalecer la educación en las escuelas de negocios, apuntando hacia un cambio en los programas y contenidos del curricular educacional de la gestión. De lo anterior se deduce que existe una preocupación por el impacto en los temas de negocios que la ética presenta, en ámbitos diversos, como la responsabilidad de los negocios en sociedad (ETHOS, 2003); liderazgo ético; la toma de decisiones; (OECD, 2003; BEDICKS y ARRUDA, 2003) gobiernos corporativos. 
Es esencial que estos sectores requieran un cambio en la actitud de entender que toda participación se reduce a una colaboración de dos términos, que a falta de uno, afectan la dinámica en conjunto. Por ejemplo, promover confianza y respeto en un ambiente laboral trae mejorías económicas e incurren en disminución de los costos, al haber más voluntad de participación y menor operativos de vigilancia en las operaciones de producción (PERLES, 2000). Además de traer resultados sumamente exitosos al traer mejorías al ambiente laboral. Si bien existe un interés como tendencia constante en la mayoría de los seres humanos lo cual puede ser moralmente lamentable, es necesario indicar que hay un cierto altruismo egoísta involuntario - búsqueda del bien estar social - debido a que esta actitud juega un doble rol de sostenible inconsistencia. Al mismo tiempo que se deben aceptar a los individuos tal como ellos son, con todos los defectos, el comportamiento altruista posee su propio interés en un intercambio sea este voluntario o negociado.

\section{Aspectos Conclusivos}

Resumiendo, si el interés es gobernado por una serie de motivaciones de valores y éste en la interacción entre individuos se representan por medio de normas que serán válidas en la medida que los valores son una opción que hace a las personas tener miras al logro de un fin. La moral está asociada a los fines, los referentes comportamentales individuales y el resultado de la interacción de lo colectivo (hábitos, costumbres, lo cotidiano, etc.) organizado en un sistema que se procesa por actividades están en el discurso de los juicios de valor.

Lo anterior se ajusta a buscar causas a las respuestas que conductas que se consideran incorrectas para uma situación que establece como normativas simbólicas de indicar lo que está bien o mal. Para efecto de sistematizar un proceso de actividades y establecer un sistema que incorpora normas se desarrolló un modelo inductivo (cuadro1) que propone entender el sentido que conlleva el discurso conclusivo de este trabajo que buscamos argumentar a través de un modelo de convergencias de campos organizacionales.

\section{Cuadro 1}

Modelo Convergente Conceptual Firma, Habitus y Ética

\begin{tabular}{|l|l|l|}
\hline Formación de Habitus & Integridad personal en la Empresa & Formación Ética (Universidad) \\
\hline $\begin{array}{l}\text { 1.-Aprender de los códigos } \\
\text { simbólicos, principalmente } \\
\text { aquellos sobre comportamiento } \\
\text { íntegro de las personas para la } \\
\text { sociedad. }\end{array}$ & $\begin{array}{l}\text { 1.- La empresa como otras } \\
\text { instituciones tienen el gran } \\
\text { objetivo (misión) de contribuir al } \\
\text { bien común. }\end{array}$ & $\begin{array}{l}\text { 1.-La Universidad debe cubrir la } \\
\text { formación del ser humano en } \\
\text { diferentes dimensiones devisión } \\
\text { en un proyecto etico-politico: } \\
\text { natural, intelectual, moral, estética } \\
\text { y religiosa. }\end{array}$ \\
$\begin{array}{l}\text { 2.-La integridad de una cultura } \\
\text { cocialmente responsiva, que } \\
\text { interpersonales sólidas para el } \\
\text { bien común. }\end{array}$ & $\begin{array}{l}\text { 2.- Bien común es aquello que } \\
\text { contribuye al común desarrollo } \\
\text { humano. La empresa debe asumir } \\
\text { su papel en la construcción de }\end{array}$ & $\begin{array}{l}\text { 2.- de socialización del estudiante, } \\
\text { sensibilidad, habilidades artísticas, } \\
\text { contribuir al desarrollo moral y } \\
\text { espíritu del pensamiento crítico. }\end{array}$ \\
$\begin{array}{l}\text { individuos impiden que la } \\
\text { asunción obstáculos deteriore la } \\
\text { comunicación. }\end{array}$ & $\begin{array}{l}\text { 3.- Los agentes, ante presiones } \\
\text { económicas de poder, son } \\
\text { capaces de dar respuestas } \\
\text { consistentes coherentes con su } \\
\text { personalidad. }\end{array}$ & $\begin{array}{l}\text { 3.- Exponer argumentación } \\
\text { /contra argumentación, } \\
\text { experiencia estética, aptitud y } \\
\text { actitud moral, experiencias que } \\
\text { estimulan y afinan el } \\
\text { reputación genera confianza y }\end{array}$ \\
\hline
\end{tabular}


Cont. Cuadro 1

\begin{tabular}{|l|l|l|}
\hline $\begin{array}{l}\text { lealtad en proyectos de } \\
\text { cooperación, desarrollo de } \\
\text { políticas de integración social no } \\
\text { garantiza lo suficiente, no habrá } \\
\text { resultados si intención de acción, } \\
\text { de palabras a los hechos. }\end{array}$ & $\begin{array}{l}\text { compromiso social de la actividad } \\
\text { empresarial. La industria debe } \\
\text { incorporar a su agenda temas de } \\
\text { responsabilidades directiva. }\end{array}$ & $\begin{array}{l}\text { 4.-Desarrollar competencias para } \\
\text { mostrar los cursos de significación } \\
\text { cultural y moral de contenidos de } \\
\text { la docencia, fundamental que los } \\
\text { estudiantes no sólo aprendan a } \\
\text { comportarse bien, sino a dar razón } \\
\text { de su comportamiento. }\end{array}$ \\
\hline
\end{tabular}

Fuente: Elaborado por el autor.

Este análisis se abre a un tipo de diálogo constructivista y no hacia un debate como tal, es decir, discursa a la manera de un diálogo como base del método de trabajo para discernir sobre las fronteras entre los campos y sus problemas así como las partes que se encuentran en permanente conflicto. Tal ensayo indagó sobre la naturaleza de los componentes que se introducen en una relación de funciones tríadicas de convergencia en un marco de campos sociales económicos como producto de juegos de intereses que se encuentran en continium de permanentes conflictos.

\section{Referencias}

AACSB (Association to Advance Collegiate Schools of Business). Ethics Education in Business School. Report of theEthics Education Task Force to AACSB International's Board of Directors. Tampa, Florida, 2004.

.Why Management Education Matters: its Impact on Individuals, Organizations, and Society. A Report from an AACSB International Task Force of the Committee on Issues in Management Education. Tampa, Florida. 2005

BEDICKS, H; ARRUDA, M. C. Business ethics and corporate governance in Latin America. White Paper on Corporate Governance in Latin America. OECD - Centre for Cooperation with Non-Members - Emerging Economies Transition, 2003. 9 p.

BOCLIN, R. Avaliação de Docentes do Ensino Superior: Um Estudo de Caso. Ensaio: aval. pol. públ. Educ., Rio de Janeiro, v. 12, n. 45, p. 959-980, Out./Dez. 2004.

BOURDIEU, P. Campo intelectual y proyecto creador. In: POUILLON, J. et al (Eds.). Problemas del Estructuralismo. México: Siglo XXI, 1983.

Coisas Ditas. São Paulo: Brasiliense. 1990.

Razões Práticas: sobre a teoria da ação.Campinas: Papirus, 1996.

A distinção: crítica social do julgamento. São Paulo: Edusp, 2007.

O poder simbólico. Rio de Janeiro: Bertrand Brasil, 2010.

; CHAMBOREDON, J. C.; PASSERON, J. C. O ofício do sociólogo. Petrópolis: Vozes, 1991.

; WACQUANT, L. J. D. An invitation to reflexive sociology. Chicago: The University of Chicago Press, 1992.

BURIASCO, R. L. C. Algumas considerações sobre avaliação educacional. Avaliação Educacional, v. 22, p. 155-178, 2000 .

CALDERON, A. I. Universidades mercantis: a institucionalização do mercado universitário em questão. São Paulo em Perspectiva, v. 14, n. 1, p. 61-72, 2000. 
Repensando o papel da universidade. Revista de Administração de Empresas, v. 44, n. 2, p. 104-108, 2004.

CHIA, R. Teaching paradigm shifting in management education: university business schools and the entrepreneurial imagination. Journal of Management Studies, v. 33, n. 4, p. 409- 428, 2000.

COHEN, J. R.; PANT, L, W.; SHARP, D. J. Project Earnings Manipulation: An Ethics Case Based on Agency Theory. Issues in Accounting Education, v. 15, n. 1, February 2000.

CONILL, J. Globalización y ética económica. Papeles de Ética, Economía y Dirección, n. 5, 2000.

ETHOS (Instituto Ethos de Empresas e Responsabilidade Social). Formulação e Implantação de Código de Ética em Empresas. Documento Reflexões e Sugestões. Agosto, 2000. Disponible en: <www.ethos.org.br>. Acesso em: Janeiro 2013.

Práticas de Responsabilidade Social das Empresas Relação entre os Princípios do Global Compact e Indicadores Ethos de Responsabilidade Social Empresarial. Carmen Weingrill (Coord.). São Paulo: Instituto Ethos, 2003.

FISCHER, T. M. D. A difusão do conhecimento sobre organizações e gestão no Brasil: seis propostas de ensino para o decênio 2000/2010. Revista de Administração Contemporânea, v. 5, n. spe, p. 123-139, 2001.

FREEMAN, E. Strategic Management: A Stakeholder Approach. New York, NY: Basic Books, 1982.

FREITAS, A.; FISCHER, T. Programa de Capacitação Docente em Administração (PCDA). Cad. EBAPE.BR, v. 5, n. Edição Especial, p. 1-2, 2007.

GAA, J. The Ethical Foundations of Public Accounting. Vancouver, BC: CGA-Canada Research Foundation, 1994.

GLASSON, J. The widening local and regional development impacts of the modern universities - a tale of two cities (and north-south perspectives). Local Economy, v. 18, n. 1, p. 21-37, 2003.

GRAMANI, M. C. A influência da qualidade na atratividade de instituições de ensino superior com capital aberto. Ensaio: aval. pol. públ. Educ., Rio de Janeiro, v. 16, n. 60, p. 437-454, Jul./Set. 2008.

GRAMSCI, A. Maquiavel, a Política e o Estado Moderno. Rio de Janeiro: Civilização Brasileira, 1989.

INEP (Instituto Nacional de Estudos e Pesquisa Educacional). Censo da Educação Superior 2004. Brasília, 2005.

JENSEN, M. C. Self-interest, altruism, incentives, \& agency theory. Foundations of Organizational Strategy, Harvard: Harvard University Press, 1998.

A Theory of the Firm: Governance, Residual Claims and Organizational Forms. Harvard: Harvard University Press, 2000. Disponible en: <http://hupress.harvard.edu/catalog/JENTHF.html>. Acesso en: Marzo 2013.

; MECKLING, W. H. The nature of man. Journal of Applied Corporate Finance, v. 7, n. 2, p. 4-19, 1994.

JONES, T. Ethical decision making by individuals in organizations: An issue contingent model. Academyof Management Review, v. 16, n. 2, p. 366-395, 1991.

JUNQUEIRA, L. A noção de representação social na Sociologia contemporânea. Estudos de Sociologia, Araraquara, v. 18/19, p. 145-161, 2005.

KOHLBERG, L. Essays on Moral Development. The Psychology of Moral Development. v. 2. San Francisco, CA: Harper \& Row, 1984.

LAHIRE, B. O Sucesso escolar nos meios populares: as razões do improvável. São Paulo: Ática, 1997.

MACINTYRE, A. After Ethics. Notre Dame: University of Notre Dame Press, 1984. 
MARTINS, C. B. A reforma universitária de 1968 e a abertura para o ensino superior privado no Brasil. Educ. Soc., Campinas, v. 30, n. 106, p. 15-35, Jan./Abr. 2009.

MARTINS, R. C. Avaliação crítica de uma experiência de ensino aprendizagem. Estudos de Psicologia, PUC Campinas, v. 16, n. 2, p. 54-64, 1999.

MEEK, V. L. Use of the Market in the Transformation of Australian Higher Education. In: GÓMEZ, R. R. (Org.). Reformas en los Sistemas Nacionales de Educación Superior. La Coruña, Es: Netbiblo, 2002. 149-178 p.

MINTZBERG, H.; GOSLING, J. Educando administradores além das fronteiras. Revista de Administração de Empresas, v. 43, n. 2, p. 29-43. 2003.

MOLLIS, M. La geopolítica de las reformas de la Educación Superior: el Norte da créditos, el Sur se "acredita". In: RODRIGUEZ GÓMEZ, R. (Org.). Reformas en los Sistemas Nacionales de Educación Superior. La Coruña, Es: Netbiblo, 2002. 321-358 p.

NICOLINI, A. Qual será o futuro das fábricas de administradores. Revista de Administração de Empresas, v. 43, n. 2, p. 44-54, 2003.

NORONHA, G. V.; ROCHA, L. B. S. Elias e Bourdieu: para uma sociologia histórica, ou seria uma história sociológica? Revista Habitus, IFCS/UFRJ, RJ, v. 5, n. 1, p. 47-58, Marzo 2008.

NOSELLA, P. Ética e pesquisa. Educ. Soc., Campinas, v. 29, n. 102, p. 255-273, Jan./Abr. 2008.

NUNES, E; De CARVALHO, M. Ensino universitário, corporação e profissão: paradoxos e dilemas brasileiros. Sociologias, Porto Alegre, v. 9, n. 17, p. 190-215, Jan./Jun.2007.

OCDE. White Paper on Corporate Governance in Latin America. OECD - Centre for Co-operation with NonMembers - Emerging Economies Transition.2003. Disponible en: <www.oecd.org/daf/corporate-affairs/>. Acceso en: Febrero 2013.

OLIVEIRA, F. B.; SAUERBRONN, F. F. Trajetória, desafios e tendências no ensino superior de administração e administração pública no Brasil: uma breve contribuição. Rev. Adm. Pública, Rio de Janeiro, v. 41, n. Edição Especial, p. 149-70, 2007.

OLIVEIRA, K. L.; DOS SANTOS, A. A. Avaliação da aprendizagem na universidade. Psicologia Escolar e Educacional, v. 9, n. 1, p. 37-46, 2005.

OROZCO, L. E. Fundamentos éticos de una política de la solidaridad. Galeras de Administración, Universidad de los Andes, Bogotá, v. 4, p. 1-38 Septiembre, 2005.

PALADINO, M; DELBEJUH, P.; DEL BOSCO, P. Integridad: respuesta superadora a los dilemas éticos del hombre de empresa. Cuad. Difus, v. 10, p. 18-19, 2005.

PAULA, A. P.; RODRIGUES, M. A. Pedagogia crítica no ensino da administração: desafios e possibilidades. Revista de Administração de Empresas, v. 46, n. Edição Especial, p. 10-22, 2006.

PERLES, G.; SANTIAGO, M. Ética y liderazgo empresarial: Una complementariedad necesaria. Papeles de Ética, Economía y Dirección, n. 5, 2000.

PINCOFFS, E. Quandaries and Virtues: Against Reductivism in Ethics. Lawrence, KS: University Press of Kansas, 1986.

PIOTTO, D. C. Trajetórias escolares prolongadas nas camadas populares. Cadernos de Pesquisa, v. 38, n. 135, p. 701727,2008

REST, J. Moral Development: Advances in Research and Theory. New York: Praeger, 1986. 
ROBINSON, C.; ADAMS, N. Unlocking the Potential: The Role of Universities in Pursuing Regeneration and Promoting Sustainable Communities. Local Economy, v. 23, n. 4, p. 277-289, November 2008.

SANTOS, A. F.; MOLINAR, M. O. O ethos da empresa na prática docente das universidades Corporativas. VII Seminário Redestrado - Nuevas Regulaciones en América Latina; Buenos Aires. 3, 4 y 5 de Julio 2008.

SCHUGURENSKY, D. Autonomía, heteronomía, y los dilemas de la educación superior en la transición al siglo XXI: el caso de Canadá. In: GÓMEZ, R. R. (Org.). Reformas en los Sistemas Nacionales de Educación Superior. LaCoruña: Netbiblo, 2002. 109-148 p.

SEVERINO, A. J. O ensino superior brasileiro: novas configurações e velhos desafios. Educar. n. 31.Curitiba: Editora UFPR, 2008. 73-89 p.

SGUISSARDI, V. Fundações Privadas na Universidade Pública - a quem interessam? Avaliação, Campinas, v. 7, n. 4, p. 43-72, 2002a.

Educação Superior no Limiar do Novo Século - traços internacionais e marcas domésticas. Revista Diálogo Educacional, v. 3, n. 7, 121-144, 2002b. (digitalizado).

SILVA JR., J. R.; SGUISSARDI, V. As novas faces da educação superior no Brasil: reforma do Estado e mudanças na produção. São Paulo: Cortez/CDAPHIFAN, 2001.

SOLOMON, R, C. La ética de los negocios. In: SINGER, P. (Ed.). Compendio de Ética. Madrid: Alianza Editorial, 1995. Cap. 31, 483-498 p.

WEBER, M. El político y el científico. México: Preponía Editores, 1981.

WOOD JR., T.; PAULA, A. P. O fenômeno dos MPAs brasileiros: hibridismo, diversidade e tensões. Revista de Administração de Empresas, v. 44, n. 1, p. 116-129, 2004. 